\title{
Association between rs1049673 polymorphism in CD36 and premature coronary heart disease
}

\author{
J.J. Che, Y.X. Shao, G.P. Li
}

Tianjin Key Laboratory of Ionic-Molecular Function of Cardiovascular Disease, Department of Cardiology, Tianjin Institute of Cardiology, Second Hospital of Tianjin Medical University, Tianjin, China

Corresponding author: J.J. Che

E-mail: jingjinche@126.com

Genet. Mol. Res. 13 (3): 7708-7717 (2014)

Received May 17, 2013

Accepted January 27, 2014

Published September 26, 2014

DOI http://dx.doi.org/10.4238/2014.September.26.8

\begin{abstract}
Risk factors for premature coronary heart disease in China can be multiple; we investigated Chinese Han patients with premature coronary heart disease and a possible association with CD36 polymorphism at rs1049673, rs7755, and rs321159 sites. Outpatients were recruited according to chest X-ray coronary arteriography results; they were divided into two groups: early coronary artery lesions (premature coronary heart disease group, test group) and a control group. Coronary arteriography and laboratory blood examinations were conducted to analyze risk factors for coronary heart disease and CD36 polymorphisms. Seventy nine test and 56 control group patients were recruited. Compared with the control, the test groups had a significantly higher proportion of male patients, smoking, diabetes and metabolic syndromes, significantly higher levels of TG, LDL-C, ox-LDL, WBC, UA, FBG, and significantly lower levels of HDL-C. For rs1049673, rs7755, and rs321159 sites, patients with premature coronary heart disease have family genetic predisposition at high LDL-C level with GA, AA, and TT genotypes. Unconditional logistic regression analysis showed that gender, diabetes, high TG, LDL-C level and C carriers
\end{abstract}


of rs1049673 significantly affected risk for premature coronary heart disease.

Key word: Coronary heart disease; Risk factors; CD36; rs1049673; rs7755; rs321159

\section{INTRODUCTION}

Coronary heart disease (CHD) is an epidemic characterized by premature onset and high mortality. The World Health Organization (WHO) reports that more than 70\% of coronary deaths occur in subjects older than 70 in North America and Western Europe and that the mortality of the young has increased in recent years (Ford and Capewell 2007; O'Flaherty et al., 2008; Bertuccio et al., 2011). Risk factors of premature CHD could be multiple, ranging from social, economic, psychological, lifestyle (such as smoking, sedentary lifestyle, improper diet), and biological (abnormal lipids, hypertension, diabetes, and obesity), and genetic factors such as mutations at specific chromosomal locations and single nucleotide polymorphisms (SNPs) have also been implicated.

Many risk factors of coronary heart disease (CHD) have been reported (Grammer et al., 2011; Rai et al., 2008; Waterworth et al., 2011), while the association between the polymorphism of scavenger receptors (SR) and CHD has been rarely reported. Recent studies showed that SR constitutes the main part of the foam cell, an important factor indicating the beginning and development of atherosclerosis (Glass and Witztum 2001; Libby, 2002). CD36, a member of the class B scavenger receptor family, is a receptor of natural lipoproteins, including low-density lipoproteins (LDL), high-density lipoproteins (HDL) and very low-density lipoproteins (VLDL). Besides, when combined with denatured lipids, it can lead to the formation of foam cells (Calvo et al., 1998; Thorne et al., 2007). A number of studies have indicated that the overexpression or polymorphism of CD36 is associated with insulin resistance, metabolic syndrome, and serum triglyceride and free fatty acid levels (Ma et al., 2004; Corpeleijn et al., 2006; Love-Gregory et al., 2008).

CD36 on the platelet surface is the bridge to lipid metabolism disorders and thrombosis (Nergiz-Unal et al., 2011). Our early studies showed that metabolic syndrome plays a pivotal role in premature CHD among Chinese patients (Che et al., 2011). In this study, we recruited Chinese Han patients with premature CHD and studied the association with CD36 polymorphism at the rs1049673, rs7755, and rs321159 sites.

\section{MATERIAL AND METHODS}

\section{Patients}

All patients were recruited from the Department of Cardiology, the Second Hospital of Tianjin Medical University between March 2009 and March 2011. These patients had symptoms of chest pain or suspected coronary artery disease, and underwent a coronary angiography after being evaluated by their attending physician.

These patients were divided into two groups according to their coronary angiography result, the criteria of the recruited patients are shown in Figure 1. The study was approved and registered by our hospital's ethics committee; all subjects signed a written informed consent form. All works were undertaken following the provisions of the Declaration of Helsinki. 


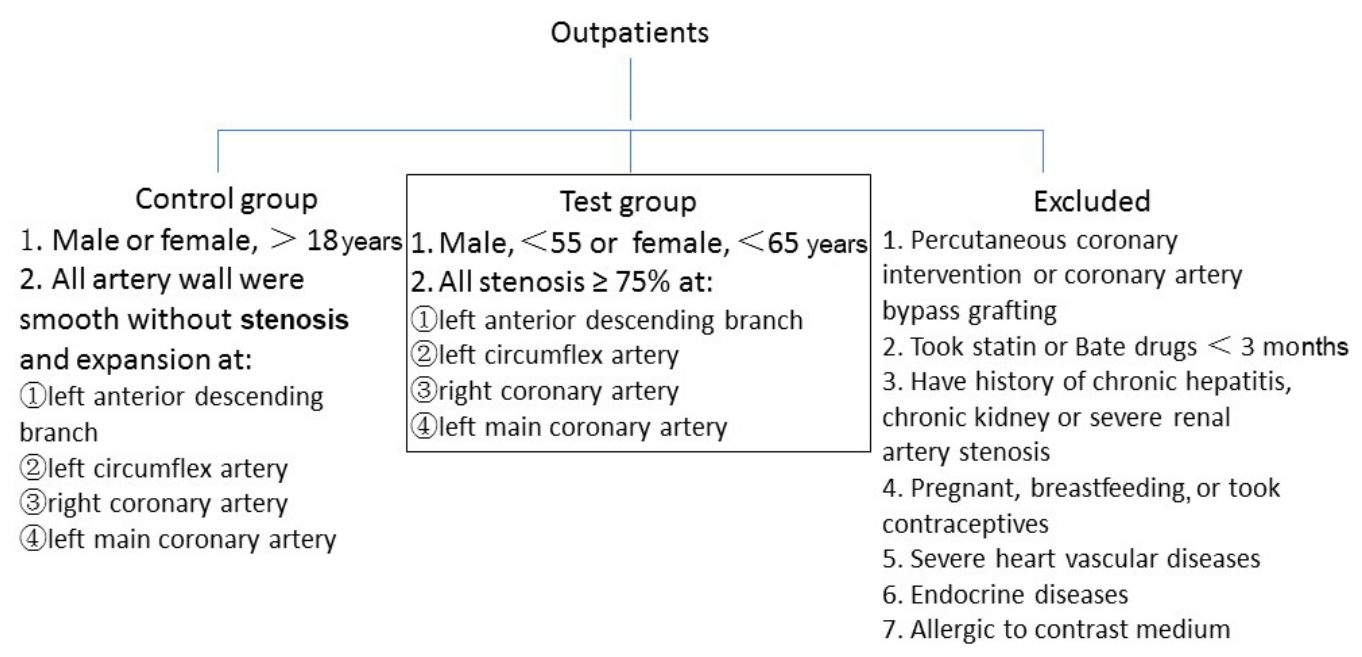

Figure 1. Patient screening process.

\section{Coronary arteriography}

Coronary angiography was performed using the standard Judkins technique (Judkins, 1967). Coronary angiograms were visually evaluated by two experienced observers according to the clinical review process. Localization and percent luminal diameter reduction were documented for the left main coronary artery branch, left anterior descending branch, left circumflex artery, and right coronary artery with stenosis. The main branches such as diagonal branch and obtuse marginal branch were classified as related main artery. Coronary angiograms were analyzed by computer-assisted quantitative coronary angiography (QCA). CHD was defined as more than $1(\geq)$ atherosclerotic plaque in a major coronary artery ( $\geq 1.5 \mathrm{~mm}$ lumen diameter) causing $\geq 50 \%$ luminal diameter stenosis by the QCA test.

\section{Clinical data collection and diagnostic criteria}

All participants' body mass index (BMI) was calculated. The detail record included gender, age, history of high blood pressure, diabetes mellitus (DM), smoking history, premature CHD family history. The standard diagnosis is shown in Table 1.

\section{Laboratory examination}

Peripheral venous blood samples were obtained from participants in the morning, where they were asked to fast at least $8 \mathrm{~h}$. Routine blood tests were performed, including hepatorenal function, fasting blood glucose (FBG), triglycerides (TG), total cholesterol (TC), LDL-C, HDL-C, VLDL-C, apoA1, apoB and plasma fibrinogen. An enzyme-linked immunosorbent assay (ELISA) kit (Calbiochem, San Diego, CA, USA) was used to determine oxidized LDL (ox-LDL) level in plasma according to manufacturer instructions. 


\begin{tabular}{lll}
\multicolumn{2}{l}{ Table 1. Primary diagnosis of premature coronary heart disease $(\mathrm{CHD})$} & and control group patients. \\
\hline Definition & Evaluation index & Threshold value \\
\hline Overweight & BMI & $\geq 25.0 \mathrm{~kg} / \mathrm{m}^{2}$ \\
Diabetes mellitus & Related symptom plus plasma glucose & $\geq 11.1 \mathrm{mM}$ \\
& $\begin{array}{l}\text { Fasting blood-glucose } \\
\text { Blood sugar at sugar tolerance test }(2 \mathrm{~h})\end{array}$ & $\geq 7.0 \mathrm{mM}$ \\
& Clear DM history & \\
Smoking & Smoke at least once/day & $1 / 1 \mathrm{mM}$ \\
& Continuous smoking & $>1$ year \\
Premature CHD family history & Direct relative has premature CHD & Male $\leq 55$ \\
Drinking & Alcohol intaking & Female $\leq 65$ \\
& Continuous drinking & $\geq 25 \mathrm{~g} / \mathrm{day}$ \\
& TC & $\geq 100 \mathrm{~g} / \mathrm{week}$ \\
High TC level & TG & $>1$ year \\
High TG level & HDL-C & $>1.70 \mathrm{mM}$ \\
Low HDL-C level & LDL-C & $>1.04 \mathrm{mM}$ \\
High LDL-C level & $>3.37 \mathrm{mM}$ \\
\hline
\end{tabular}

$\mathrm{TC}=$ total cholesterol; TG = triglycerides; HDL-C = high-density lipoproteins cholesterol; LDL-C = low-density lipoproteins cholesterol; $\mathrm{BMI}=$ body mass index; $\mathrm{DM}=$ diabetes mellitus.

\section{Measurement of CD36 gene polymorphism}

After $10 \mathrm{~mL}$ blood were drawn from the cubital vein and anti-coagulated, the white blood cells were separated and immediately stored at $-80^{\circ} \mathrm{C}$. Extraction of genomic DNA and PCR amplification were conducted according to the instructions of the related reagent suppliers. The template contained three SNPs (rs1049673, rs7755, rs321159) of CD36 DNA. After the extension and purification, the products were analyzed by matrix-assisted laser desorptionionization time-of-flight mass spectrometry (MALDI-TOFMS).

\section{Statistical method}

Data were analyzed by the SPSS11.5 software. Quantitative data are expressed as means \pm SE. Comparison between two groups with normal distribution was done by an independent sample $t$ test, and with non-normal distribution by the Mann-Whitney $\mathrm{U}$ test. Risk factors were analyzed by multi-factor logistic regression analysis. $\mathrm{P}<0.05$ was considered to be statistically significant.

\section{RESULTS}

Seventy-nine premature CHD patients and 56 control patients were recruited in our study. The basic clinical characteristics and routine blood test results (Table 2) showed no statistical significance in premature family history, hypertension rate, BMI and TC, VLDL-C, apoprotein A1, platelets, fibrinogen, blood urea nitrogen, serum albumin, total bilirubin, and conjugated bilirubin levels in the test and control groups.

The test group had a higher proportion of male patients (68 vs 41\%), smoking (59 vs 39\%), diabetes (39 vs 13\%) and metabolic syndrome (apoprotein B: $0.86 \pm 0.15$ vs $0.76 \pm$ $0.09 \mathrm{mM})$ compared with controls $(\mathrm{P}<0.05)$. The levels of TG $(2.36 \pm 1.88 v s 1.66 \pm 1.19$ $\mathrm{mM})$, LDL-C $(3.20 \pm 0.80 v s 2.83 \pm 0.58 \mathrm{mM})$, ox-LDL ( 634 vs $561 \mu \mathrm{g} / \mathrm{L}), \mathrm{UA}(376.6 \pm 116.9$ vs $312.0 \pm 100.2 \mathrm{mM})$, FBG $(7.2 \pm 2.8$ vs $6.3 \pm 3.1 \mathrm{mM})$ were remarkably higher in the test group $(\mathrm{P}<0.05)$, while the level of HDL-C $(1.08 \pm 0.18 v s 1.31 \pm 0.28 \mathrm{mM})$ was lower in the test group $(\mathrm{P}<0.05)$. 
Table 2. Basic clinical characters and blood routine examination comparison result in two groups.

\begin{tabular}{|c|c|c|c|}
\hline Variant & Control $(\mathrm{N}=56)$ & Test group $(\mathrm{N}=79)$ & $P$ value \\
\hline Age, years & $61.2 \pm 10.4$ & $53.6 \pm 6.9$ & 0.001 \\
\hline Male $(\mathrm{N}, \%)$ & $23(41)$ & $54(68)$ & 0.002 \\
\hline Premature FH (N, \%) & $3(5)$ & $6(8)$ & 0.608 \\
\hline Hypertension (N, \%) & $30(54)$ & $54(68)$ & 0.081 \\
\hline Diabetes ( $N, \%)$ & $7(13)$ & $31(39)$ & 0.009 \\
\hline Smoker (N, \%) & $22(39)$ & $47(59)$ & 0.021 \\
\hline Body mass index $\left(\mathrm{kg} / \mathrm{m}^{2}\right)$ & $24.1 \pm 3.1$ & $25.1 \pm 3.1$ & 0.073 \\
\hline Triglyceride (mM) & $1.66 \pm 1.19$ & $2.36 \pm 1.88$ & 0.016 \\
\hline Total cholesterol (mM) & $4.91 \pm 0.80$ & $5.10 \pm 1.07$ & 0.272 \\
\hline LDL cholesterol (mM) & $2.83 \pm 0.58$ & $3.20 \pm 0.80$ & 0.005 \\
\hline HDL cholesterol (mM) & $1.31 \pm 0.28$ & $1.08 \pm 0.18$ & $<0.001$ \\
\hline VLDL cholesterol (mM) & $0.78 \pm 0.41$ & $0.88 \pm 0.41$ & 0.401 \\
\hline Apoprotein A1 (mM) & $1.24 \pm 0.17$ & $1.20 \pm 0.16$ & 0.429 \\
\hline Apoprotein B (mM) & $0.76 \pm 0.09$ & $0.86 \pm 0.15$ & 0.008 \\
\hline Oxidized LDL $(\mu \mathrm{g} / \mathrm{L})$ & $561(440-660)$ & $634(512-844)$ & $0.002 *$ \\
\hline Platelet (x 10 $\left.10^{12} / \mathrm{L}\right)$ & $220 \pm 57$ & $226 \pm 48$ & $0.558^{*}$ \\
\hline Fibrinogen $(\mathrm{g} / \mathrm{L})$ & $2.9 \pm 0.6$ & $2.9 \pm 0.5$ & 0.858 \\
\hline Blood urea nitrogen $(\mathrm{mM})$ & $5.6 \pm 2.0$ & $6.6 \pm 5.8$ & 0.217 \\
\hline Serum creatinine $(\mu \mathrm{M})$ & $69.9 \pm 20.1$ & $80.0 \pm 37.7$ & $0.003 *$ \\
\hline Uric acid $(\mu \mathrm{M})$ & $312.0 \pm 100.2$ & $376.6 \pm 116.9$ & $0.001 *$ \\
\hline Serum albumin $(g / L)$ & $40.0 \pm 3.0$ & $39.0 \pm 3.2$ & 0.079 \\
\hline Total bilirubin $(\mu \mathrm{M})$ & $11.5 \pm 4.0$ & $12.5 \pm 5.4$ & 0.255 \\
\hline Conjugated bilirubin $(\mu \mathrm{M})$ & $2.6 \pm 1.3$ & $2.8 \pm 1.6$ & 0.402 \\
\hline Fasting blood glucose (mM) & $6.3 \pm 3.1$ & $7.2 \pm 2.8$ & 0.042 \\
\hline
\end{tabular}

*Result of nonparametric Mann-Whitney test, other quantitative data was compared by independent sample $t$ test.

\section{rs1049673, rs7755, and rs321159 polymorphism analysis}

For rs 1049673 , G carriers had a higher proportion of premature CHD (60.7\% in control group and $51.3 \%$ in test group) than did C carriers (39.3\% in control group and $48.7 \%$ in test group, $\mathrm{P}=0.030)$. For rs 7755 , A carriers had a higher proportion of premature CHD $(60.9 \%$ in controls and $51.3 \%$ in test group) than did $\mathrm{G}$ carriers (39.1\% in controls and $48.7 \%$ in test group, $\mathrm{P}=0.032)$. For $\mathrm{rs} 321159$, T carriers had a higher proportion of premature $\mathrm{CHD}(66.7 \%$ in controls and $72.8 \%$ in test group) than did $\mathrm{G}$ carriers (33.3\% in control and $27.2 \%$ in test group), but the difference was not statistically significant $(\mathrm{P}=0.065)$. All these $\mathrm{SNP}$ results are shown in Table 3.

As we can see from Table 4 (detailed data not shown), for rs1049673, CC carriers had a higher level of LDL-C than GG carriers, which showed family genetic predisposition with statistical significance $(\mathrm{P}=0.049)$, which means patients with premature $\mathrm{CHD}$ all had the $\mathrm{CC}$ or GC genotype. For rs7755, AA carriers had a higher level of LDL-C than GG carriers with statistical significance $(\mathrm{P}=0.05)$. It also showed a family genetic predisposition, which means patients with premature CHD all had the CC or GC genotype. For rs321159, TT carriers had the highest level of LDL-C, while GG carriers had the lowest level $(\mathrm{P}=0.010)$, and the other genotype showed no significant difference.

\section{Independent predictors of premature CHD}

To evaluate the level of the mutation of rs1049673 in the CD36 gene among CHD risk factors, binary logistic regression analysis was used. The three main coronary artery vessel lesions were considered as dependent variables, and gender, hypertension, DM, hyperuricemia, high level of TG and LDL-C, high and low level of HDL-C, positive C carriers 
at rs 1049673, and positive $\mathrm{G}$ carriers at rs7755 were considered independent variables. After unconditional logistic regression analysis, the results (Table 5) showed gender, DM, high TG symptom, high LDL-C symptom and C carriers of rs 1049673 all could predic premature CHD remarkably.

Table 3. Composition of genotypes at rs1049673, rs7755, and rs3211956 sites.

\begin{tabular}{|c|c|c|c|c|}
\hline Site & Genotype & Control $(\mathrm{N}=56)$ & Test group $(\mathrm{N}=79)$ & $P$ value \\
\hline \multirow[t]{5}{*}{ rs1049673 } & GG & $37.5 \%(21)$ & $25.3 \%(20)$ & 0.281 \\
\hline & GC & $46.4 \%(26)$ & $51.9 \%(41)$ & \\
\hline & $\mathrm{CC}$ & $16.1 \%(9)$ & $22.8 \%(18)$ & \\
\hline & $\mathrm{G}$ & $60.7 \%(68)$ & $51.3 \%(81)$ & 0.030 \\
\hline & $\mathrm{C}$ & $39.3 \%(44)$ & $48.7 \%(77)$ & \\
\hline \multirow[t]{5}{*}{ rs 7755} & $\mathrm{AA}$ & $38.2 \%(21)$ & $25.3 \%(20)$ & 0.260 \\
\hline & GA & $45.5 \%(25)$ & $51.9 \%(41)$ & \\
\hline & GG & $16.3 \%(9)$ & $22.8 \%(18)$ & \\
\hline & A & $60.9 \%(67)$ & $51.3 \%(81)$ & 0.032 \\
\hline & G & $39.1 \%(43)$ & $48.7 \%(77)$ & \\
\hline \multirow{5}{*}{ rs3211956 } & TT & $40.7 \%(22)$ & $55.7 \%(44)$ & 0.127 \\
\hline & GT & $51.9 \%(28)$ & $34.2 \%(27)$ & \\
\hline & GG & $7.4 \%(4)$ & $10.1 \%(8)$ & \\
\hline & $\mathrm{T}$ & $66.7 \%(72)$ & $72.8 \%(115)$ & 0.065 \\
\hline & G & $33.3 \%(36)$ & $27.2 \%(43)$ & \\
\hline
\end{tabular}

Table 4. Distribution and clinical character of rs1049673, rs7755, and rs3211956 polymorphism in CD36.

\begin{tabular}{|c|c|c|c|c|c|}
\hline & $\mathrm{N}$ & PFH (\%) & $\mathrm{P}$ & LDL cholesterol (mM) & $\mathrm{P}$ \\
\hline \multicolumn{6}{|c|}{ rs1049673 } \\
\hline GG & 41 & 0 & 0.03 & $2.9 \pm 0.7$ & 0.049 \\
\hline GC & 67 & 11 & & $3.0 \pm 0.8$ & \\
\hline $\mathrm{CC}$ & 27 & 7 & & $3.3 \pm 0.7$ & \\
\hline \multicolumn{6}{|c|}{ rs 7755} \\
\hline GG & 41 & 0 & 0.03 & $2.89 \pm 0.69$ & 0.05 \\
\hline GA & 66 & 11 & & $3.03 \pm 0.75$ & \\
\hline $\mathrm{AA}$ & 27 & 7 & & $3.34 \pm 0.73$ & \\
\hline \multicolumn{6}{|c|}{ rs3211956 } \\
\hline GG & 12 & 0 & 0.102 & $2.69 \pm 0.70$ & 0.01 \\
\hline GT & 55 & 4 & & $2.91 \pm 0.62$ & \\
\hline TT & 66 & 11 & & $3.23 \pm 0.78$ & \\
\hline
\end{tabular}

$\mathrm{PFH}=$ Premature family history of coronary heart disease; LDL = low-density lipoproteins. Quantitative data is compared by ANOVA test.

Table 5. Binary gradually logistic regression analysis of independent predictor in premature coronary heart disease.

\begin{tabular}{|c|c|c|c|c|}
\hline Variant & $\mathrm{B}$ & SE & Wald & $\mathrm{P}$ \\
\hline Gender & 1.595 & 0.469 & 11.557 & 0.001 \\
\hline rs1049673 dominant inheritance* & 1.117 & 0.468 & 6.314 & 0.012 \\
\hline Diabetes & 2.082 & 0.612 & 11.584 & 0.001 \\
\hline High TG level & 0.996 & 0.440 & 5.130 & 0.024 \\
\hline High LDL-C level & 1.201 & 0.519 & 5.357 & 0.021 \\
\hline Constant & 4.607 & 0.903 & 26.034 & $<0.001$ \\
\hline
\end{tabular}

The following factors were excluded from logistic regression equation: positive G carriers at rs7755, hypertension, hyperuricemia, low-high density cholesterol symptom. The value of enumeration data is as follows: gender " 0 " represents female, "1" represents male. The condition of coronary " 0 " represents normal, "1" represents lesion. Diabetes, high TG symptom, high LDL-C symptom, smoking history " 0 " represents no, "1" represents existence. *According to the genetic model of rs 1049673 (GG or GC genotype is 0, CC genotype is 1). According to the genetic model of rs 7755 (AA genotype is 0, AG or GG genotype is 1). 


\section{DISCUSSION}

Studies have shown that conventional cardiovascular risk factors differ markedly between premature and non-premature CAD (Genest et al., 1991; Reibis et al., 2012). Reibis et al. (2012) reported dyslipidemia, as well as family smoking history, were dominant risk factors in a young group of premature $\mathrm{CAD}$. Hence, we excluded the patients receiving lipid-lowering statins or other lipid-lowering drugs and patients who received percutaneous coronary intervention and bypass surgery. The relationship between the standard LDL-C and coronary events were confirmed (LaRosa et al., 2005; Reiner et al., 2011; Robinson and Stone, 2006), whereas the relation between hypertriglyceridemia and coronary heart disease (CHD) is still controversial (Cullen, 2000). Previous experience in young post-infarction patients indicates that young patients are characterized by mixed lipid disorders, particularly those resulting from disturbances in the metabolism of triglyceride-rich lipoproteins (Tornvall et al., 1991). In our study, hypertriglyceridemia and DM were proven to be an essential risk factor of premature CAD in Chinese Han people. Especially at an age less than 65, males are more likely to have CAD compared with females (Vaidya et al., 2007; Hoffmann et al., 2008). Our study confirmed this result.

As for rs1049673 in the CD 36 gene, Vaidya et al. (2007) demonstrated that the Framingham risk equation underestimated total incident 10-year CAD events in individuals with a sibling history of premature $\mathrm{CAD}$, and the excess risk observed could be attributed to genetic and other susceptibility factors. The polymorphism of CD36 has been reported to be associated with glucose metabolism disorders as well as triglyceride and free fatty acid levels. Ma et al. (2004) investigated the polymorphism of 21 allele sites in CD36 in 585 native non-diabetic Caucasian patients. As shown by the results, there was a strong relation between the polymorphism of rs 1049673 as well as two other sites and free fatty acid level $(\mathrm{P}=0.02)$. More specifically, the rs 1049673 polymorphism revealed a stronger association with male patients $(\mathrm{P}=0.008)$. $\mathrm{G}$ homozygous male patients had a higher free fatty acid level than males who were $\mathrm{C}$ homozygous, while they were similar in triglyceride and HDL levels. In addition, although for rs1049673, the level of serum LDL was higher in the CC genotype carriers than those with the GG genotype, the gap was not statistically significant (Ma et al., 2004). Noel et al. (2010) investigated 1178 Puerto Ricans living in Boston. They confirmed that SNPs of CD36 were remarkably associated with metabolic syndrome in diabetes and systolic hypertension in the high incidence group. In detection of six alleles in the site, the SNP rs 1049673 showed the strongest link with metabolic syndrome, in which the possibility of metabolic syndrome among minimum allele carriers increased (odds ratio $=1.89$ ). We found that CC carriers of rs 1049673 had a higher level of serum LDL than did GG carriers in Chinese Han people, while the association between SNPs in this gene and the level of serum TG in diabetes is still unknown. More importantly, we initially proved that the $\mathrm{C}$ carriers of rs 1049673 had a higher opportunity to have premature CHD than did G carriers. This may be due to the high level of serum LDL in C carriers, which is also the main characteristic of blood fat in premature CHD. Thus, we believe that the SNP rs 1049673 in $\mathrm{CD} 36$ is an independent predictor of premature CHD.

As for rs7755, this CD36 SNPs was studied previously, and phenotypes were shown to be related to lipid metabolism (Corpeleijn et al., 2006; Ma et al., 2004). We initially proved that different genotypes at rs 7755 site had a strong link with the level of LDL, and that A carriers had a higher proportion of premature CHD as well as a higher level of LDL than did G carriers. However, the proportion of diabetes and BMI, FBG and triglyceride levels between 
different genotypes of rs7755 did not differ. This indicates that these SNPs influence mainly LDL metabolism rather than the level of blood glucose and triglycerides at the incidence of premature CHD. Heni et al. (2011) demonstrated that the CD36 SNP rs3211956 significantly is associated with BMI. Another study showed the SNP in CD36 was significantly $(\mathrm{P}=0.04)$ more common in patients with acute MI (minor allele frequency $10.5 \%$ ) than patients with stable exertional angina (minor allele frequency 8.0\%) (Knowles et al., 2007). Our study confirmed that different genotypes of rs3211956 are associated with the level of LDL-C and that $\mathrm{T}$ carriers have a higher risk of premature CHD but with no statistical significance.

In general, the polymorphisms of rs 1049673, rs7755, and rs3211956 are all associated with the level of LDL-C, but the related mechanism is still unknown. CD36 is not only an essential receptor combining the long-chain fatty acid and denatured LDL, but also participates in the reverse transport of HDL to the liver. Cholesterol is reverse transported to the liver from the surrounding tissues by HDL, which combines with related receptors on liver cell surface and is then transferred into the liver cell by endocytosis. Finally it becomes the bile acid and is expelled outside. Brundert et al. (2011) confirmed that compared with wild-type mice, knockdown CD36 gene mice absorb less $\left[{ }^{3} \mathrm{H}\right]$ and $\left[{ }^{125} \mathrm{I}\right]$ markers in the liver. Besides, cultured primary hepatocytes totally unable to absorb HDL in vitro. On the contrary, CD36 expressed in adenovirus-mediated cells show recovery of HDL uptake ability. Thus, the polymorphism of CD36 could influence the level of plasma LDL-C by the reverse transport of cholesterol. Among the SNPS studied, rs 1049673 and rs 7755 show little relevance to premature CHD, and rs 1049673 is an independent risk factor of premature CHD. The polymorphism of rs 1049673 and rs7755 in CD36 could affect other factors besides plasma LDL-C.

Meanwhile, effects of the three polymorphisms on plasma ox-LDL were involved in our study. Studies showed that normal LDL is not able to induce foam cell formation. But modified LDLs, especially the oxidized LDLs, can induce foam cell formation from macrophages. Atherosclerotic lesions are characterized by the accumulation of ox-LDL and the infiltration of macrophages and T cells (Binder et al., 2002; Hansson and Hermansson, 2011). The course of LDL oxidization mainly happens on the vessel wall, but recently ox-LDLs were reported to be detected in peripheral blood (Johnston et al., 2006). We also observed that the level of serum ox-LDL in premature CHD patients was higher than in controls. The main way to eliminate ox-LDL is phagocytosis of macrophages by SR, whose main member is CD36 (Park et al., 2012; Picard et al., 2010). So, the polymorphism of the CD36 gene could influence the level of ox-LDL. The polymorphisms of rs1049673, rs7755, and rs3211956 in CD36 significantly impact the level of LDL-C in peripheral blood, but cannot affect the level of oxLDL. The possible reasons may be: 1) the difference between different levels of ox-LDL in patients from the same group is very large, making it hard to assess the impact of ox-LDL in different genotypes and thereby needing a larger sample size; 2) the polymorphism of CD36 may influence the content of ox-LDL in the walls of blood vessels rather than in peripheral blood.

Our data were only based on a small-scale case-control study. We hope that larger prospective studies in the future might explore the role of CD36 variants in the development of this condition.

\section{REFERENCES}

Bertuccio P, Levi F, Lucchini F, Chatenoud L, et al. (2011). Coronary heart disease and cerebrovascular disease mortality 
in young adults: recent trends in Europe. Eur. J. Cardiovasc. Prev. Rehabil. 18: 627-634.

Binder CJ, Chang MK, Shaw PX, Miller YI, et al. (2002). Innate and acquired immunity in atherogenesis. Nat. Med. 8: 1218-1226.

Brundert M, Heeren J, Merkel M, Carambia A, et al. (2011). Scavenger receptor CD36 mediates uptake of high density lipoproteins in mice and by cultured cells. J. Lipid. Res. 52: 745-758.

Calvo D, Gomez-Coronado D, Suarez Y, Lasuncion MA, et al. (1998). Human CD36 is a high affinity receptor for the native lipoproteins HDL, LDL, and VLDL. J. Lipid. Res. 39: 777-788.

Che J, Li G, Wang W, Li Q, et al. (2011). Serum autoantibodies against human oxidized low-density lipoproteins are inversely associated with severity of coronary stenotic lesions calculated by Gensini score. Cardiol. J. 18: 364-370.

Corpeleijn E, van der Kallen CJ, Kruijshoop M, Magagnin MG, et al. (2006). Direct association of a promoter polymorphism in the CD36/FAT fatty acid transporter gene with Type 2 diabetes mellitus and insulin resistance. Diabet. Med. 23: 907-911.

Cullen P (2000). Evidence that triglycerides are an independent coronary heart disease risk factor. Am. J. Cardiol. 86: 943-949.

Ford ES and Capewell S (2007). Coronary heart disease mortality among young adults in the U.S. from 1980 through 2002: concealed leveling of mortality rates. J. Am. Coll. Cardiol. 50: 2128-2132.

Genest JJ, McNamara JR, Salem DN and Schaefer EJ (1991). Prevalence of risk factors in men with premature coronary artery disease. Am. J. Cardiol. 67: 1185-1189.

Glass CK and Witztum JL (2001). Atherosclerosis. The road ahead. Cell 104: 503-516.

Grammer TB, Hoffmann MM, Renner W, Kleber ME, et al. (2011). Apolipoprotein E genotypes, circulating C-reactive protein and angiographic coronary artery disease: the Ludwigshafen Risk and Cardiovascular Health Study. Atherosclerosis 215: 487-493.

Hansson GK and Hermansson A (2011). The immune system in atherosclerosis. Nat. Immunol. 12: 204-212.

Heni M, Mussig K, Machicao F, Machann J, et al. (2011). Variants in the CD36 gene locus determine whole-body adiposity, but have no independent effect on insulin sensitivity. Obesity 19: 1004-1009.

Hoffmann U, Massaro JM, Fox CS, Manders E, et al. (2008). Defining normal distributions of coronary artery calcium in women and men (from the Framingham Heart Study). Am. J. Cardiol. 102: 1136-41, 1141.

Johnston N, Jernberg T, Lagerqvist B, Siegbahn A, et al. (2006). Improved identification of patients with coronary artery disease by the use of new lipid and lipoprotein biomarkers. Am. J. Cardiol. 97: 640-645.

Judkins MP (1967). Selective coronary arteriography. I. A percutaneous transfemoral technic. Radiology 89: 815-824.

Knowles JW, Wang H, Itakura H, Southwick A, et al. (2007). Association of polymorphisms in platelet and hemostasis system genes with acute myocardial infarction. Am. Heart J. 154: 1052-1058.

LaRosa JC, Grundy SM, Waters DD, Shear C, et al. (2005). Intensive lipid lowering with atorvastatin in patients with stable coronary disease. N. Engl. J. Med. 352: 1425-1435.

Libby P (2002). Inflammation in atherosclerosis. Nature 420: 868-874.

Love-Gregory L, Sherva R, Sun L, Wasson J, et al. (2008). Variants in the CD36 gene associate with the metabolic syndrome and high-density lipoprotein cholesterol. Hum. Mol. Genet. 17: 1695-1704.

Ma X, Bacci S, Mlynarski W, Gottardo L, et al. (2004). A common haplotype at the CD36 locus is associated with high free fatty acid levels and increased cardiovascular risk in Caucasians. Hum. Mol. Genet. 13: 2197-2205.

Nergiz-Unal R, Lamers MM, Van KR, Luiken JJ, et al. (2011). Signaling role of CD36 in platelet activation and thrombus formation on immobilized thrombospondin or oxidized low-density lipoprotein. J. Thromb. Haemost. 9: 1835-1846.

Noel SE, Lai CQ, Mattei J, Parnell LD, et al. (2010). Variants of the CD36 gene and metabolic syndrome in Boston Puerto Rican adults. Atherosclerosis 211: 210-215.

O'Flaherty M, Ford E, Allender S, Scarborough P, et al. (2008). Coronary heart disease trends in England and Wales from 1984 to 2004: concealed levelling of mortality rates among young adults. Heart 94: 178-181.

Park YM, Drazba JA, Vasanji A, Egelhoff T, et al. (2012). Oxidized LDL/CD36 interaction induces loss of cell polarity and inhibits macrophage locomotion. Mol. Biol. Cell 23: 3057-3068.

Picard E, Houssier M, Bujold K, Sapieha P, et al. (2010). CD36 plays an important role in the clearance of oxLDL and associated age-dependent sub-retinal deposits. Aging 2: 981-989.

Rai TS, Khullar M, Sehrawat BS, Ahuja M, et al. (2008). Synergistic effect between apolipoprotein E and apolipoprotein A1 gene polymorphisms in the risk for coronary artery disease. Mol. Cell Biochem. 313: 139-146.

Reibis R, Treszl A, Wegscheider K, Bestehorn K, et al. (2012). Disparity in risk factor pattern in premature versus lateonset coronary artery disease: a survey of 15,381 patients. Vasc. Health Risk Manag. 8: 473-481.

Reiner Z, Catapano AL, De BG, Graham I, et al. (2011). ESC/EAS Guidelines for the management of dyslipidaemias: the Task Force for the management of dyslipidaemias of the European Society of Cardiology (ESC) and the European Atherosclerosis Society (EAS). Eur. Heart J. 32: 1769-1818. 
Robinson JG and Stone NJ (2006). Identifying patients for aggressive cholesterol lowering: the risk curve concept. $A m$. J. Cardiol. 98: 1405-1408.

Thorne RF, Mhaidat NM, Ralston KJ and Burns GF (2007). CD36 is a receptor for oxidized high density lipoprotein: implications for the development of atherosclerosis. FEBS Lett. 581: 1227-1232.

Tornvall P, Karpe F, Carlson LA and Hamsten A (1991). Relationships of low density lipoprotein subfractions to angiographically defined coronary artery disease in young survivors of myocardial infarction. Atherosclerosis 90 : 67-80.

Vaidya D, Yanek LR, Moy TF, Pearson TA, et al. (2007). Incidence of coronary artery disease in siblings of patients with premature coronary artery disease: 10 years of follow-up. Am. J. Cardiol. 100: 1410-1415.

Waterworth DM, Ricketts SL, Song K, Chen L, et al. (2010). Genetic variants influencing circulating lipid levels and risk of coronary artery disease. Arterioscler. Thromb. Vasc. Biol. 30: 2264-2276. 